\title{
ON DILATION EQUATIONS AND THE HÖLDER CONTINUITY OF THE DE RHAM FUNCTIONS
}

\author{
by YIBIAO PAN
}

(Received 15 January, 1993)

\begin{abstract}
We use a simple approximation method to prove the Hölder continuity of the generalized de Rham functions.
\end{abstract}

1. Consider the following dilatation equation

$$
\begin{aligned}
f(x)= & f(3 x)+\left(\frac{1}{2}-\alpha\right)(f(3 x+1)+f(3 x-1)) \\
& +\left(\frac{1}{2}+\alpha\right)(f(3 x+2)+f(3 x-2)),
\end{aligned}
$$

where $|\alpha|<1 / 2$. Suppose that $f$ is an integrable solution of (1); then $f$ must satisfy

$$
\hat{f}(\xi)=p_{\alpha}(\xi / 3) \hat{f}(\xi / 3),
$$

where $\hat{f}(\xi)=\int e^{i x \xi} f(x) d x$ is the Fourier transform of $f$, and

$$
\begin{aligned}
p_{\alpha}(\xi) & =\left[1+\left(\frac{1}{2}-\alpha\right)\left(e^{i \xi}+e^{-i \xi}\right)+\left(\frac{1}{2}+\alpha\right)\left(e^{2 i \xi}+e^{-2 i \xi}\right)\right] / 3 \\
& =e^{-2 i \xi}\left(\frac{1+e^{i \xi}+e^{2 i \xi}}{3}\right)\left[\left(\frac{1}{2}+\alpha\right)-2 \alpha e^{i \xi}+\left(\frac{1}{2}+\alpha\right) e^{2 i \xi}\right],
\end{aligned}
$$

which immediately leads to

$$
\hat{f}(\xi)=\hat{f}(0) \prod_{n=1}^{\infty} p_{\alpha}\left(\frac{\xi}{3^{n}}\right)
$$

It turns out that (1) does have integrable solutions. (By (4), such a solution is unique up to multiplication by a constant.) When $\alpha=1 / 6$, the function which is integrable, satisfies (1) and $\hat{f}(0)=1$, is called the de Rham function. For other values of $\alpha$, such functions are called the generalized de Rham functions.

In [1] and [2], Daubechies and Lagarias studied the existence and properties of solutions of general dilation equations. One of their results, obtained by using the time-domain method, shows that the generalized de Rham functions are supported on $[-1,1]$, and are Hölder continuous, with Hölder exponent

$$
\gamma_{\alpha}=\min \left[-\ln \left|\frac{1}{2}+\alpha\right|,-\ln |2 \alpha|\right] / \ln 3 .
$$

It is stated in [1] that it is not known how to prove the continuity of the de Rham function directly from (3). The purpose of this note is to provide a proof of their result using precisely (3). For results on more general dilation equations, we refer the reader to [1] and [2]. 
2. Let $\alpha \in\left(-\frac{1}{2}, \frac{1}{2}\right)$. The result mentioned above is stated as the following theorem.

THEOREM. There exists an integrable function $f$ which satisfies (1) and $\hat{f}(0)=1$. The function $f$ is Hölder continuous with exponent $\gamma_{\alpha}$; i.e. there is an $A_{\alpha}>0$ such that

$$
|f(x)-f(y)| \leq A_{\alpha}|x-y|^{\gamma_{\alpha}},
$$

for $x, y \in \mathbf{R}$.

We now present our proof. Let

$$
q_{\alpha}(\xi)=\left(\frac{1}{2}+\alpha\right)-2 \alpha e^{i \xi}+\left(\frac{1}{2}+\alpha\right) e^{2 i \xi}
$$

By (3) and the equation

$$
\prod_{n=1}^{\infty} e^{-2 i \xi / 3^{n}}\left(\frac{1+e^{i \xi / 3^{n}}+e^{2 i \xi / 3^{n}}}{3}\right)=\frac{1-e^{-i \xi}}{i \xi},
$$

which is the Fourier transform of $\chi_{(-1,0]}$, we are led to the following sequence of functions $\left\{f_{n}\right\}_{n=0}^{\infty}$, defined recursively by

$$
\begin{gathered}
f_{0}(x)=\chi_{(-1,0]}(x) \\
f_{n}(x)=\left(\frac{1}{2}+\alpha\right) f_{n-1}(x)-2 \alpha f_{n-1}\left(x-\frac{1}{3^{n}}\right)+\left(\frac{1}{2}+\alpha\right) f_{n-1}\left(x-\frac{2}{3^{n}}\right)
\end{gathered}
$$

Let $k \in \mathbf{N}, j \in \mathbf{Z}$. Clearly, the function $f_{k}$ assumes a constant value on every interval of the form $\left((j-1) / 3^{k}, j / 3^{k}\right]$, and $\operatorname{supp}\left(f_{k}\right) \subset[-1,1]$. Let $v_{k, j}$ be the value of $f_{k}$ on $\left((j-1) / 3^{k}, j / 3^{k}\right]$. We find that

Therefore

$$
\begin{aligned}
v_{k+1,3 j-2} & =\left(\frac{1}{2}+\alpha\right) v_{k, j}+\left(\frac{1}{2}-\alpha\right) v_{k, j-1}, \\
v_{k+1,3 k-1} & =\left(\frac{1}{2}-\alpha\right) v_{k, j}+\left(\frac{1}{2}+\alpha\right) v_{k, j-1}, \\
v_{k+1,3 j} & =v_{k, j}
\end{aligned}
$$

$$
\sup _{x}\left|f_{k+1}(x)-f_{k}(x)\right| \leq \sup _{j}\left|v_{k, j}-v_{k, j-1}\right| .
$$

On the other hand, we have

$$
\begin{aligned}
v_{k+1,3 j}-v_{k+1,3 j-1} & =\left(\frac{1}{2}+\alpha\right)\left(v_{k, j}-v_{k, j-1}\right), \\
v_{k+1,3 j-1}-v_{k+1,3 j-2} & =(-2 \alpha)\left(v_{k, j}-v_{k, j-1}\right), \\
v_{k+1,3 j-2}-v_{k+1,3 j-3} & =\left(\frac{1}{2}+\alpha\right)\left(v_{k, j}-v_{k, j-1}\right) .
\end{aligned}
$$

Let $\beta=\max \left\{\left|\frac{1}{2}+\alpha\right|,|2 \alpha|\right\}$. Using (11)-(13), we get

$$
\sup _{j}\left|v_{k, j}-v_{k, j-1}\right| \leq \beta^{k}
$$

Since $0 \leq \beta<1$, the sequence $\left\{f_{n}\right\}$ converges pointwise to a function $f$; i.e. $f=\lim f_{n}$. Clearly $\operatorname{supp}(f) \subset[-1,1]$. We now show that $f$ satisfies (1) and (5).

Take $x, y$ which satisfy

$$
3^{-N-1} \leq|x-y|<3^{-N}
$$


for some integer $N>0$. Then

$$
\begin{aligned}
|f(x)-f(y)| & \leq\left|f(x)-f_{N}(x)\right|+\left|f_{N}(x)-f_{N}(y)\right|+\left|f_{N}(y)-f(y)\right| \\
& \leq \sum_{k=N}^{\infty}\left|f_{k+1}(x)-f_{k}(x)\right|+\sum_{k=N}^{\infty}\left|f_{k+1}(y)-f_{k}(y)\right|+\sup _{j}\left|v_{N, j}-v_{N, j-1}\right| \\
& \leq 2 \sum_{k=N}^{\infty} \beta^{k}+\beta^{N} \leq A_{\alpha} \beta^{N} \leq A_{\alpha}|x-y|^{\gamma_{\alpha}} .
\end{aligned}
$$

To show that $f$ satisfies (1), we observe that $0 \leq \lim \left\|f-f_{n}\right\|_{1} \leq 2 \lim \left\|f-f_{n}\right\|_{\infty}=0$,

$$
\hat{f}_{n}(\xi)=q_{\alpha}\left(\xi / 3^{n}\right) \hat{f}_{n-1}(\xi)=\ldots=\frac{1-e^{-i \xi}}{i \xi} \prod_{k=1}^{n} q_{\alpha}\left(\xi / 3^{k}\right)
$$

and

$$
q_{\alpha}\left(\xi / 3^{n+1}\right) \hat{f}_{n}(\xi)=p_{\alpha}(\xi / 3) \hat{f}_{n}(\xi / 3)
$$

Let $n \rightarrow \infty$; we find that

$$
\hat{f}(\xi)=p_{\alpha}(\xi / 3) \hat{f}(\xi / 3)
$$

This shows that $f$ is indeed a continuous, integrable solution of (1). Finally we show that (5) becomes false if $\gamma_{\alpha}$ is replaced by any larger exponent.

From (10) it is clear that $f_{m}(x)=f_{k}(x)$, if $x=j .3^{-k}$, and $m \geq k$. Hence, for such $x$, $f(x)=f_{k}(x)$. Let

$$
x_{k}=-3^{-k}\left(\sum_{j=0}^{k-1} 3^{j}\right), \quad y_{k}=-3^{-k}\left(\sum_{j=0}^{k-1} 3^{j}+1\right), \quad z_{k}=-1+3^{-k}
$$

Then $\left|x_{k}-y_{k}\right|=\left|z_{k}-(-1)\right|=3^{-k}$. By (11)-(13), we get

$$
\begin{aligned}
\left|f\left(x_{k}\right)-f\left(y_{k}\right)\right| & =\left|f_{k}\left(x_{k}\right)-f_{k}\left(y_{k}\right)\right| \\
& =|2 \alpha|\left|f_{k-1}\left(x_{k-1}\right)-f_{k-1}\left(y_{k-1}\right)\right|=\ldots=|2 \alpha|^{k},
\end{aligned}
$$

and $\left|f\left(z_{k}\right)-f(-1)\right|=|1 / 2+\alpha|^{k}$, which show that (5) cannot hold if $\gamma_{\alpha}$ is replaced by any larger number.

\section{REFERENCES}

1. I. Daubechies and J. Lagarias, Two-scale difference equations I. Existence and global regularity of solutions, SIAM J. Anal. 22 (1991), 1388-1410.

2. I. Daubechies and J. Lagarias, Two-scale difference equations II. Local regularity, infinite product of matrices and fractals, to appear.

Department of Mathematics and Statistics

UNIVERSITY OF PITTSBURGH

PitTsburgh

Pensyluania 15260

U.S.A. 